\title{
UNA DESIGUALDAD TRIGONOMETRICA A TRAVÉS DE LA INDUCCIÓN MATEMÁTICA
}

RICARDO CEDEÑO T. Y OLGA LUCIA YUSTES H. Universidad Surcolombiana

A raíz del Seminario de investigación que realizan los estudiantes del Programa de Matemáticas y Física de la Universidad Surcolombiana encontramos un método utilizado por Cauchy. Aqui lo hemos adaptado para demostrar una desigualdad trigonométrica por Inducción Matemática.

Si $\alpha$ y $\beta$ son ángulos que satisfacen las condiciones $0 \leq \alpha \leq 180^{\circ}$ y $0 \leq \beta \leq 1800$, entonces

$$
\operatorname{sen} \alpha+\operatorname{sen} \beta \leq 2 \operatorname{sen}\left(\frac{\alpha+\beta}{2}\right)
$$

Tenemos la igualdad en el caso que $\alpha=\beta$. Para demostrar esto podemos inicialmente mostrar que

$\operatorname{sen} \alpha+\operatorname{sen} \beta=2 \operatorname{sen}\left(\frac{\alpha+\beta}{2}\right) \cos \left(\frac{\alpha-\beta}{2}\right)$

En efecto:

$\operatorname{sen} \alpha+\operatorname{sen} \beta=\operatorname{sen}\left(\frac{\alpha+\beta}{2}+\frac{\alpha-\beta}{2}\right)+\operatorname{sen}\left(\frac{\alpha+\beta}{2}-\frac{\alpha-\beta}{2}\right)=\operatorname{sen}\left(\frac{\alpha+\beta}{2}\right) \cos \left(\frac{\alpha+\beta}{2}\right)+$ $\operatorname{sen}\left(\frac{\alpha-\beta}{2}\right) \cos \left(\frac{\alpha+\beta}{2}\right)+\operatorname{sen}\left(\frac{\alpha+\beta}{2}\right) \cos \left(\frac{\alpha-\beta}{2}\right)-\operatorname{sen}\left(\frac{\alpha-\beta}{2}\right) \cos \left(\frac{\alpha+\beta}{2}\right)=$ $2 \operatorname{sen}\left(\frac{\alpha+\beta}{2}\right) \cos \left(\frac{\alpha-\beta}{2}\right)$. Ahora,

$\alpha-\beta$ satisface $-180^{\circ} \leq \alpha-\beta \leq 180^{\circ}$ esto lleva a que $\cos (\underline{(\alpha-\beta)})$ es positivo o es cero, 2

con valor máximo 1 , así tenemos que sen $\alpha+\operatorname{sen} \beta=2 \operatorname{sen} \frac{(\alpha+\beta)}{2} \cos \left(\frac{\alpha-\beta}{2} \leq\right.$ 


\section{$2 \operatorname{sen}(\alpha+\beta)$.}

2

Es natural pensar en la generalización de este resultado. Intuitivamente, tenemos que $\alpha_{1}, \alpha_{2} \ldots, \alpha_{n}$ son ángulos que satisfacen las condiciones $0 \leq a \alpha_{1} \leq 180^{\circ}$ para todo $j=1,2, \ldots, n$ entonces

$\operatorname{sen} \alpha_{1}+\operatorname{sen} \alpha_{2}+\ldots+\operatorname{sen} \alpha_{n} \leq n \operatorname{sen} n(\underline{\alpha 1+\alpha 2+\ldots+\alpha n})$, la igualdad

ocurre Si $\alpha_{1}=\alpha_{2}=\ldots=\alpha_{n}$

Para demostrar esta corazonada, lo trataremos desarrorando una técnica empleada por Cauchy para tratar demostraciónes por Inducción Matemática.

Si llamamos a Pn, la función proposicional $\operatorname{sen} \alpha_{1}+\operatorname{sen} \alpha_{2}+\ldots+\operatorname{sen} \alpha_{n} \leq n$ sen $n$ $(\alpha 1+\alpha 2+\ldots+\alpha n)$, sabemos ya que $P 1$ y $P 2$ son verdaderas. 2

Vamos a suponer que $P_{n}$, es válida para cualquier entero $n \geq 3$, y trataremos de establecer la veracidad de $P_{n}-1$.

Sea a $\alpha_{1}+\alpha_{2}+\ldots+\alpha_{n}-1, n-1$ ángulos no todos iguales, $y$ definamos a $\alpha_{n} \operatorname{como} \alpha_{n}=\frac{(\alpha 1+\alpha 2+\ldots+\alpha n-1)}{n-1}=$ observemos que $\alpha_{1}+\alpha_{2}+\ldots+\alpha_{n-1}+\alpha_{n}=\left(\alpha_{1}+\alpha_{2}+\ldots\right.$ $\left.+\alpha_{n-1}\right)+\frac{(\alpha 1+\alpha 2+\ldots+\alpha n-1)}{n-1}=(\alpha 1+\alpha 2+\ldots+\alpha n-1)\left(1+\frac{n}{n-1}\right)=\left(\frac{n}{n-1}\right)(\alpha 1+\alpha 2+\ldots+\alpha n-1)=$ $\left(\frac{n}{n-1}\right)(n-1) \alpha n=n \alpha_{n}$. Así que sen $\alpha 1+\ldots+\operatorname{sen}_{n-1}+\operatorname{sen} \alpha_{n} \leq n \operatorname{sen} n \frac{(\alpha 1+\ldots+\alpha n)}{n}$ implica que $\operatorname{sen} \alpha 1+\operatorname{sen} \alpha 2+\ldots+\operatorname{sen} \alpha_{n-1}+\operatorname{sen} \alpha_{n} \leq n \operatorname{sen} \alpha_{n}=(n-1) \operatorname{sen} \frac{(\alpha 1+\ldots+\alpha n)}{n-1}$ Ahora a partir de suponer que $P n$ es verdadera veamos que $P 2 n$ también lo es.

Consideremos $\alpha_{1}+\alpha_{2}+\ldots+\alpha_{2 n}$ donde los $\alpha_{1}$ son ángulos cualesquiera, no todos iguales, satisfaciendo $0 \leq \alpha_{1} \leq 180^{\circ}$ entonces tenemos: $\operatorname{sen} \alpha 1+\operatorname{sen} \alpha 2+\ldots+\operatorname{sen} \alpha_{n}$

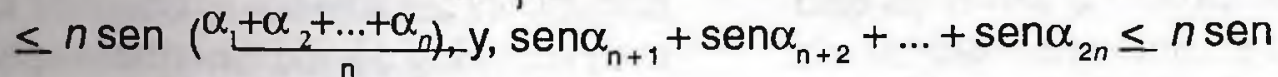
$\left(\frac{\operatorname{sen} a n+1+\operatorname{sen} a n+2+\ldots+\operatorname{sen} \alpha 2 n}{n}\right)$, luego $\operatorname{sen} \alpha_{1}+\operatorname{sen} \alpha_{2}+\ldots+\operatorname{sen} \alpha_{2 n} \leq n \operatorname{sen} n\left(\frac{\alpha_{1}+\ldots+\alpha n}{n}\right)+$ $n \operatorname{sen} n\left(\frac{\alpha n+1+\alpha 2 n}{n}\right)=n\left[\operatorname{sen}\left(\frac{\alpha 1++\alpha n}{n}\right)+\operatorname{sen}\left(\frac{\alpha n+1+\ldots+\alpha 2 n}{n}\right)\right] \leq 2 n$

$\left[\operatorname{sen}\left(\frac{\frac{\alpha 1+\ldots+\alpha n}{n}+\frac{\alpha n+1+\ldots+\alpha 2 n}{n}}{2}\right)\right] 2 n \operatorname{sen}\left(\frac{\alpha 1+\ldots+\alpha 2 n}{n}\right)$.

Este resultado lo podemos enunciar como:

Teorema. Si $\alpha_{1}+\alpha_{2}+\ldots,+\alpha_{n}$ Son ángulos que satisfacen las condiciones $0 \leq \alpha_{j} \leq$ $180^{\circ}$ para todo $j=1,2, \ldots, n$ entonces: $\operatorname{sen} \alpha_{1}+\ldots+\operatorname{sen} \alpha_{n}+\leq n \operatorname{sen}\left(\frac{\alpha 1}{n}+\ldots+\alpha n\right)$ la igualdad sí y sólo sí $\alpha_{1}=\alpha_{2,}=\ldots=\alpha_{n}$. 
Es bastante interesante e ingenioso el método desarrollado por Cauchy para demostrar teoremas utilizando la Inducción Matemática.

\section{BIBLIOGRAFIA}

1. Apostol, Tom., Calculus. Volumen I, Editorial Reverté, 1.972.

2. Cedeño, R., Penagos M., La inducción en la educación secundaria, XII Coloquio Distrital de Matemáticas y Estadística, 1.995.

3. Niven, Ivan, Maxima and Minima Without Calculus, The Mathematical Association of America, 1.981.

4. Yustes, 0. L., Seminario de Investigación, Universidad Surcolombiana, 1996. DEPARTAMENTO DE MATEMATICAS, UNIVERSIDAD SURCOLOMBIANA, NEIVA - HUILA.\& 